\title{
UNIONS OF COCKCROFT TWO-COMPLEXES
}

\author{
by W. A. BOGLEY \\ (Received 11th November 1992)
}

\begin{abstract}
A combinatorial hypothesis is presented that serves as a necessary and sufficient condition for a union of connected Cockcroft two-complexes to be Cockcroft. This combinatorial hypothesis has a component that can be expressed in terms of the second homology of groups. The hypothesis is applied to the study of the third homology of groups.
\end{abstract}

1991 Mathematics subject classification: Primary 57M20, Secondary 20F05, 20 J05.

\section{Introduction}

It was observed by Cockcroft [5] that if a two-complex $K$ (that is, a two-dimensional $\mathrm{CW}$ complex) is a subcomplex of an aspherical two-complex, then for any basepoint $z \in K$, the Hurewicz map $h: \pi_{2}(K, z) \rightarrow H_{2} K$ is trivial. In other words, each spherical map $S^{2} \rightarrow K$ is homologically trivial. Two-complexes with this latter property are therefore said to be Cockcroft. In this note, necessary and sufficient conditions are given for a union $K$ of subcomplexes $K_{r}$ and $K_{s}$ to be Cockcroft. A necessary condition is that each of $K_{r}$ and $K_{s}$ be Cockcroft. An additional condition is group-theoretic; it is of interest in connection with a certain "relative Hopf formula" for third homology that was introduced in [1].

This introductory section discusses refinements and the significance of the Cockcroft property. The main result is proved in Section 2. Examples and applications to homology calculations are presented in Section 3.

The Cockcroft property has group-theoretic content. Suppose that $K$ is modeled on a presentation $\mathscr{P}=(\mathbf{x}: \mathbf{u})$ for a group $G$. For $u \in \mathbf{u}$, write $u=q^{e}$ where $e \geqq 1$ and $q$ is not a proper power. If $K$ is Cockcroft, then $q$ determines an element of order exactly $e$ in $G \cong \pi_{1} K[18,20]$. Next, there is the exact Hopf sequence $[15,16]$

$$
0 \rightarrow H_{3} G \rightarrow \mathrm{Z} \otimes_{\mathrm{zG}} \pi_{2} \mathrm{~K} \rightarrow \mathrm{H}_{2} \mathrm{~K} \rightarrow \mathrm{H}_{2} \mathrm{G} \rightarrow \mathrm{O}
$$

where the middle map is naturally induced by $h$. It follows that if $K$ is Cockcroft, then $\mathrm{H}_{2} G \cong \mathrm{H}_{2} \mathrm{~K}$ is free abelian and $\mathrm{H}_{3} G \cong \mathrm{Z} \otimes_{\mathrm{zG}} \pi_{2} \mathrm{~K}$. From a combinatorial perspective, if $K$ is finite and Cockcroft, then $\mathscr{P}$ is efficient, in the sense that the number of generators minus the number of relators is equal to the difference of the torsion-free rank of $H_{1} G$ minus the minimum number of generators for $\mathrm{H}_{2} G$ [8]. In particular, $\mathrm{K}$ has minimum Euler characteristic among all finite two-complexes with fundamental group isomorphic 
to $G$ [2]. Finally, the Cockcroft property has been used to produce lower bounds for isoperimetric functions of group presentations $[9,17]$.

Increasingly delicate versions of the Cockcroft property arise upon passage to coverings of $K$. For a subgroup $H \leqq \pi_{1} K$, the two-complex $K$ is $H$-Cockcroft if the lifted Hurewicz map $\bar{h}: \pi_{2} \mathrm{~K} \rightarrow \mathrm{H}_{2} \bar{K}$ is trivial, where $\bar{K} \rightarrow K$ is the covering corresponding to $H$. This is the same as saying that $\bar{K}$ is Cockcroft. In particular, $K$ is aspherical if and only if $K$ is $\{1\}$-Cockcroft. For $H, H^{\prime} \leqq \pi_{1} K$, if $K$ is $H$-Cockcroft and some $\pi_{1} K$-conjugate of $H$ is contained in $H^{\prime}$, then $K$ is $H^{\prime}$-Cockcroft. (This is because conjugate subgroups arise by changing basepoints in covering complexes.)

As an example, suppose that $K$ is modeled on a one-relator presentation (x:r), where $r \neq 1$ in the free group $F$ with basis $\mathbf{x}$. Write $r=q^{e}$ where $q$ is not a proper power in $F$. It follows from Lyndon's Simple Identity Theorem [19] that for a subgroup $H \leqq \pi_{1} K, K$ is $H$-Cockcroft if and only if $H$ contains each $\pi_{1} K$-congugate of $q$. The point is that the Identity Theorem can be interpreted as a description of a generating set for $\pi_{2} K$. Generalizations of this observation appear in [10].

These refined versions of the Cockcroft property first appeared in [2], and have since received considerable attention $[3,7,8,10,11,14,20,21]$. They provide an intrinsic connection between the subgroup structure of $\pi_{1} K$ and the module structure of $\pi_{2} K$. It is shown in [10] and in [14] that if $K$ is Cockcroft, then $\pi_{1} K$ contains a minimal subgroup $H$ such that $K$ is $H$-Cockcroft. These so-called threshold subgroups are studied extensively in $[10,11]$. Examples of two-complexes with nonunique thresholds are presented in [21].

\section{The main result}

Suppose that a connected two-complex $K$ is given as the union of connected subcomplexes $K_{r}$ and $K_{s}$, where $K_{r} \cap K_{s}=K^{(1)}$. Let $F=\pi_{1} K^{(1)}$ and let $R=\operatorname{ker}\left(F \rightarrow \pi_{1} K_{r}\right)$ and $S=\operatorname{ker}\left(F \rightarrow \pi_{1} K_{s}\right)$ be the kernels of the inclusion-induced maps. The subgroups of $\pi_{1} K=F / R S$ are all of the form $N / R S$, where $R S \subseteq N \leqq F$. If $A$ and $B$ are subgroups of $F$, then $[A, B]$ denotes the subgroup of $F$ generated by all commutators $[a, b]=$ $a b a^{-1} b^{-1}$, where $a \in A$ and $b \in B$.

Theorem. For a subgroup $N \leqq F$ with $R S \subseteq N, K$ is $N / R S$-Cockcroft if and only if

(i) $K_{r}$ is $N / R$-Cockcroft,

(ii) $K_{s}$ is $N / S$-Cockcroft, and

(iii) $R \cap S \subseteq[R, N] \cap[S, N]$.

Proof. Let $p: \bar{K} \rightarrow K$ be the covering of $K$ corresponding to $N / R S$. Let $p_{r}: \bar{K}_{r} \rightarrow K$, be the restriction of $p$ to $\bar{K}_{r}=p^{-1}\left(K_{r}\right) ; p_{r}$ is the covering of $K_{r}$ corresponding to $N / R$. If $K$ is $N / R S$-Cockcroft, then $\bar{K}$ is Cockcroft, and so the subcomplex $\bar{K}$, is Cockcroft. Similarly, $\bar{K}_{s}=p^{-1}\left(K_{s}\right)$ is Cockcroft. We may thus assume throughout that $K_{r}$ is $N / R$-Cockcroft and that $K_{s}$ is $N / S$-Cockcroft. The following commutative diagram has exact rows and columns. The top row is from [12, Theorem 1] (see also [4, Corollary 
3.2] and [1, Corollary 3.4]) and the middle row is from the Mayer-Vietoris homology sequence for $\bar{K}=\bar{K}_{r} \cup \bar{K}_{s}$. Exactness of the first two columns is due to Hopf $[15,16]$.

$$
\begin{aligned}
& \pi_{2} K_{r} \oplus \pi_{2} K_{s} \quad \rightarrow \quad \pi_{2} K \quad \stackrel{\eta}{\rightarrow} \frac{R \cap S}{[R, S]} \rightarrow 0 \\
& \downarrow 0 \quad \downarrow h \quad \downarrow v^{N} \\
& 0 \rightarrow \quad H_{2} \bar{K}_{r} \oplus H_{2} \bar{K}_{s} \quad \rightarrow \quad H_{2} \bar{K} \quad \rightarrow \quad H_{1} \bar{K}^{(1)} \\
& \downarrow \cong \quad \text { lonto } \\
& \mathrm{H}_{2} \mathrm{~N} / \mathrm{R} \oplus \mathrm{H}_{2} \mathrm{~N} / \mathrm{S} \rightarrow \mathrm{H}_{2} \mathrm{~N} / \mathrm{RS}
\end{aligned}
$$

As in [1, Lemma 3.1], the homomorphism $v^{N}$ can be identified with the map

$$
\frac{R \cap S}{[R, S]} \rightarrow \frac{N}{[N, N]}
$$

that is induced by the inclusion of $R \cap S$ in $N$. Applying the snake lemma, Hopf's formula for the second homology of groups shows that the bottom row of the diagram can be extended to the left and rewritten as the exact sequence

where

$$
\operatorname{ker} v^{N} \stackrel{i}{\rightarrow} \frac{R \cap[N, N]}{[R, N]} \oplus \frac{S \cap[N, N]}{[S, N]} \rightarrow \frac{R S \cap[N, N]}{[R S, N]},
$$

$$
i(w[R, S])=\left(w[R, N], w^{-1}[S, N]\right)
$$

for each $w \in R \cap S \cap[N, N]$. To see this, a description of the map $\eta$ is given in [12, p. 49] and in [1, Remarks 2.6 and 3.6]. Namely, given $w \in R \cap S$, there exist singular discs $\alpha:\left(B^{2}, S^{1}\right) \rightarrow\left(\bar{K}_{r}, \bar{K}^{(1)}\right)$ and $\beta:\left(B^{2}, S^{1}\right) \rightarrow\left(\bar{K}_{s} . \bar{K}^{(1)}\right)$ with boundaries $\left.\alpha\right|_{S^{1}}=\left.\beta\right|_{s^{1}}$ representing $w \in \pi_{1} \bar{K}^{(1)} \cong N$. One then has that

$$
[\alpha][\beta]^{-1} \in \operatorname{ker}\left(\pi_{2}\left(\bar{K}, \bar{K}^{(1)}\right) \rightarrow \pi_{1} \bar{K}^{(1)}\right) \cong \pi_{2} K
$$

and that $\eta\left([\alpha][\beta]^{-1}\right)=w[R, S]$. If $w \in R \cap S \cap[N, N]$ then the image of $[\alpha]$ under the relative Hurewicz map $\pi_{2}\left(\bar{K}_{r}, \bar{K}^{(1)}\right) \rightarrow H_{2}\left(\bar{K}_{r}, \bar{K}^{(1)}\right)$ is an element of $\operatorname{ker}\left(H_{2}\left(\bar{K}_{r}, \bar{K}^{(1)}\right) \rightarrow H_{1} \bar{K}^{(1)}\right) \cong H_{2} \bar{K}_{r}$. Further, this element projects to $w[R, N]$ under the Hopf map $\mathrm{H}_{2} \bar{K}_{r} \rightarrow(R \cap[N, N]) /[R, N]$. Similar remarks apply to $[\beta]$ and the description of $i$ follows directly.

A diagram chase now reveals that

$$
K \text { is } N / R S \text {-Cockcroft } \Leftrightarrow h=0 \Leftrightarrow v^{N}=0 \text { and } i=0 .
$$

The definitions of $v^{N}$ and $i$ thus imply that $K$ is $N / R S$-Cockcroft if and only if

$$
R \cap S \subseteq[N, N] \text { and } R \cap S \cap[N, N] \subseteq[R, N] \cap[S, N] \text {, }
$$


which is equivalent to the assertion that

$$
R \cap S \subseteq[R, N] \cap[S, N]
$$

It is worth noting that if $K$ is $N / R S$-Cockcroft, then $i=0$, and so the natural map

$$
\mathrm{H}_{2} \mathrm{~N} / \mathrm{R} \oplus \mathrm{H}_{2} \mathrm{~N} / \mathrm{S} \rightarrow \mathrm{H}_{2} \mathrm{~N} / \mathrm{RS}
$$

is injective. (Compare $[10,11]$.)

Taking $N=R S$, the theorem yields a result on asphericity of two-complexes. All notation is that of the theorem.

Corollary. $K$ is aspherical if and only if

(i) $K_{r}$ is $R S / R$-Cockcroft,

(ii) $K_{s}$ is $R S / S$-Cockcroft, and

(iii) $R \cap S \subseteq[R, R][R, S] \cap[S, S][R, S]$.

This result can also be deduced from [12, Theorem 1] and an exact sequence due to $R$. Brown [4].

\section{Examples}

The condition (iii) of the theorem is of interest in the study of the third integral homology of groups. The four-term Hopf sequence displayed in the Introduction shows that elements in third homology are the residues of homologically trivial spherical maps when the homotopy action of the fundamental group is trivialized. It is relatively easy to produce homologically trivial spherical maps in specific examples. To decide whether a spherical homotopy class survives when the fundamental group action is trivialized is more difficult; this problem involves the internal structure of $\pi_{2}$.

A combinatorial approach to this problem was introduced in [1]. Among the results there is a "relative Hopf formula" for third homology [1, Corollary 5.5] in the form of an exact sequence

$$
H_{3} F / R \oplus H_{3} F / S \stackrel{j}{\rightarrow} H_{3} F / R S \stackrel{e}{\rightarrow} \frac{[R, F] \cap[S, F]}{[R, S][F, R \cap S]} \rightarrow 0
$$

The value of this sequence begins with the fact that the homology of one-relator groups is completely understood [19]. One may think of the image of $j$ as representing "obvious" elements of $H_{3} F / R S$. Results on the kernel of $j$ appear in [6]. The image of $e$ carries the "nonobvious" elements of $H_{3} F / R S$. If $R \cap S \subseteq[R, F] \cap[S, F]$, then every element of $R \cap S$ determines an element in the image of $e$. This is relevant to the search 
for nonobvious elements in $H_{3} F / R S$ as it is easier to produce elements of $R \cap S$ than of the subgroup $[R, F] \cap[S, F]$.

As in [1, Section 6], suitable conditions on $R \cap S$ facilitate the detection of nonobvious elements of $H_{3} F / R S$. The lower central series of $F$ provides a good context for calculations. For a positive integer $n$, let $F_{n}$ denote the $n$th term of the lower central series of the free group $F$. Thus, $F_{1}=F$, and $F_{n+1}=\left[F, F_{n}\right]$. The quotient groups $F_{n} / F_{n+1}$ are free abelian, with bases determined by the basic commutators of weight $n$. (A good reference for this is $[13$, p. 149ff].) From the relative Hopf formula, it follows that if $R \cap S \subseteq F_{n}$, then there is a homomorphism

$$
e_{n}: H_{3} F / R S \rightarrow \frac{F_{n}}{[R, S] F_{n+1}}
$$

such that $e_{n} j=0$. The image of $e_{n}$ therefore detects non-obvious elements ${ }^{2} H_{3} F / R S$. Elements in the image of $e_{n}$ are represented by elements of $[R, F] \cap[S, F]$.

Proposition. Let $(\mathbf{x}: \mathbf{r}, \mathbf{s})$ be a group presentation and let $F$ be the free group with basis x. Assume that the following conditions hold.

(i) There is a positive integer $n$ such that $\mathbf{r} \cup \mathbf{s} \subseteq F_{n}$ and

(ii) the elements $u F_{n+1}(u \in \mathbf{r} \cup \mathbf{s})$ are linearly independent in the free abelian group $F_{n} / F_{n+1}$.

Let $R$ (resp. S) denote the normal closure of $\mathbf{r}$ (resp. s) in F. Then,

$$
R \cap S \subseteq[R, F] \cap[S, F] .
$$

It follows that $R \cap S \subseteq F_{n+1}$ and that the model of $(\mathrm{x}: \mathrm{r}, \mathrm{s})$ is Cockcroft.

Proof. Let $w \in R \cap S$. There exist $r_{i} \in \mathbf{r}, s_{j} \in \mathbf{S}, w_{i}, v_{j} \in F$, and $\varepsilon_{i}, \delta_{j}= \pm 1$ such that

$$
w=\prod_{i} w_{i} r_{i}^{R_{i}} w_{i}^{-1}=\prod_{j} v_{j} s_{j}^{s} v_{j}^{-1} .
$$

For $r \in \mathbf{r}$, let

$$
n_{r}=\sum_{r_{i}=r} \varepsilon_{i}
$$

and similarly define $n_{s}$ for each $s \in \mathbf{s}$. Since $\mathbf{r} \cup \mathbf{s} \subseteq F_{n}$,

$$
w F_{n+1}=\prod_{i} r_{i}^{\prime \prime} F_{n+1}=\prod_{r \in r} r^{n r} F_{n+1}
$$

and hence 


$$
\prod_{r \in r} r^{n_{r}} F_{n+1}=\prod_{s \in s} s^{n_{s}} F_{n+1}
$$

The linear independence hypothesis now implies that $n_{u}=0$ for all $u \in \mathbf{r} \cup \mathbf{s}$. Finally,

$$
w[R, F]=\prod_{i} r_{i}^{e_{i}}[R, F]=\prod_{r \in \mathrm{r}} r^{n_{r}}[R, F]=1[R, F]
$$

so $w \in[R, F]$. It follows similarly that $w \in[S, F]$. Since $R \subseteq F_{n}$, we also have that $R \cap S \subseteq F_{n+1}$. Since the model of a one-relator presentation is Cockcroft, it follows by induction, compact supports and the theorem that the model $K$ of $(x: r, s)$ is Cockcroft.

This generalizes a result from [8], which considers the case $n=1$.

For the presentation considered in the Proposition, there is the homomorphism $e_{n+1}: H_{3} F / R S \rightarrow F_{n+1} /[R, S] F_{n+2}$. Examples with $n=1$ appear in [1, Example 6.3, 6.4]. In each of the examples, the homomorphism $e_{2}$ is shown to be nontrivial.

For an example in the case $n=2$, let $F$ be the free group with basis $\{x, y, z\}$. Let $\mathbf{r}=\left\{\left[x^{a}, y\right]\right\}$ and $\mathbf{s}=\left\{\left[y^{b}, z\right],\left[z^{c}, x\right]\right\}$, where $a b c \neq 0$. Since the elements $\left[x^{a}, y\right] F_{3}$, $\left[y^{b}, z\right] F_{3},\left[z^{c}, x\right] F_{3}$ are linearly independent in $F_{2} / F_{3} \cong Z^{(3)}$, the Proposition provides that $R \cap S \subseteq[R, F] \cap[S, F] \cap F_{3}$. Since $R S \subseteq F_{2}$, we have the homomorphism

$$
e_{3}: H_{3} F / R S \rightarrow F_{3} /[R, S] F_{4}=F_{3} / F_{4} \text {. }
$$

Each element $\mu \in R \cap S$ determines an element $\mu F_{4}$ in the image of $e_{3}$. Now, it can be shown that

$$
\mu=x^{a} z^{c} x^{-a} y^{b} x^{a} y^{-b} z^{-c} y^{b} x^{-a} y^{-b} \in R \cap S
$$

For example, since $x^{a}$ centralizes all powers of $y$ modulo $R$,

$$
\mu R=x^{a} z^{c} y^{b} y^{-b} z^{-c} x^{-a} R=1 R
$$

and so $\mu \in R$. Similarly, $\mu \in S$. (It is not too difficult to produce elements of $R \cap S$ geometrically using equators of spherical pictures. See $[1,6,20]$ for discussions.) Manipulations with the commutator identities yield

$$
\begin{aligned}
\mu F_{4} & =x^{a} z^{c}\left[x^{-a}, y^{b}\right] z^{-c}\left[y^{b}, x^{-a}\right] x^{-a} F_{4} \\
& =x^{a}\left[z^{c},\left[x^{-a}, y^{b}\right]\right] x^{-a} F_{4} \\
& =[[x, y], z]^{a b c} F_{4} \in F_{3} / F_{4} .
\end{aligned}
$$

Since $[[x, y], z] F_{4}$ is contained in a basis for the free abelian group $F_{3} / F_{4}$, it follows that $\mu F_{4}$ is the image under $e_{3}$ of a nonobvious element of infinite order in $H_{3} F / R S$. 
Note that if $K$ is the model of $(x, y, z: \mathbf{r}, \mathbf{s})$, then $h: \pi_{2} K \rightarrow H_{2} K$ is trivial, although both $\pi_{2} \mathrm{~K}$ and $\mathrm{H}_{2} \mathrm{~K}$ are nontrivial.

Take $\mathbf{r}=\left\{\left[x,\left[x, y^{c}\right]\right]\right\}$ and $\mathbf{s}=\left\{\left[y,\left[x, y^{c}\right]\right]\right\}$ in the free group $F$ with basis $\{x, y\}$, where $c \neq 0$. Here, one can show that

$$
\mu=\left[x, y^{c}\right] x y^{c} x^{-1}\left[y^{c}, x\right] x\left[x, y^{c}\right] y^{-c}\left[y^{c}, x\right] x^{-1} \in R \cap S .
$$

The proposition applies with $n=3$, giving the homomorphism

$$
e_{4}: H_{3} F / R S \rightarrow F_{4} / F_{5} \text {. }
$$

Further, one finds that

$$
\mu F_{5}=[y,[x[x, y]]]^{c^{2}} F_{5},
$$

thereby detecting a non-obvious element of infinite order in $\mathrm{H}_{3} \mathrm{~F} / \mathrm{RS}$.

Huck and Rosebrock [17] have used the fact that the model of $\left(x, y:\left[x,\left[x, y^{c}\right]\right]\right.$, $\left.\left[y,\left[x, y^{c}\right]\right]\right)$ is Cockcroft to show that this presentation does not have a quadratic isoperimetric inequality.

\section{REFERENCES}

1. W. A. Bogley and M. A. Gutiérrez, Mayer-Vietoris sequences in homotopy of 2-complexes and in homology of groups, J. Pure Appl. Algebra 77 (1992), 39-65.

2. J. Brandendurg and M. Dyer, On J. H. C. Whitehead's aspherical question I, Comment. Math. Helv. 56 (1981), 431-446.

3. J. Brandenburg, M. Dyer and R. Strebel, On J. H. C. Whitehead's aspherical question II, in Low Dimensional Topology (S. Lomonaco, ed., Contemp. Math. 20, 1983), 65-78.

4. R. Brown, Coproducts of crossed $P$-modules: Applications to second homotopy groups and the homology of groups, Topology 23 (1984), 337-345.

5. W. H. Cockcroft, On two-dimensional aspherical complexes, Proc. London Math. Soc. 4 (1954), 375-384.

6. A. J. Duncan, G. Ellis and N. D. Gilbert, A Mayer-Vietoris sequence in group homology and the decomposition of relation modules (Heriott-Watt University and University College, Galway, 1992), preprint.

7. M. N. Dyer, Crossed modules and the second homotopy modules of two-complexes, in Combinatorial Group Theory and Homotopy in Low Dimensions (C. Hog-Angelloni, W. Metzler, and A. J. Sieradski, editors, London Math. Soc. Lecture Note Series, Cambridge University Press), to appear.

8. M. N. Dyer, Cockcroft 2-complexes (University of Oregon, 1993), preprint.

9. S. M. Gersten, Dehn functions and $l_{1}$-norms of finite presentations, in Algorithms and Classification in Combinatorial Group Theory (G. Baumslag and C. F. Miller III, editors, MSRI 23, 1991).

10. N. D. Gilbert and J. Howie, Threshold subgroups for Cockcroft 2-complexes (Heriot-Watt University, 1992), preprint. 
11. N. D. Gilbert and J. Howie, Cockcroft properties of graphs of 2-complexes, Proc. Roy. Soc. Edinburgh, to appear.

12. M. A. Gutierrez and J. Ratcliffe, On the second homotopy group, Quart. J. Math. Oxford (2) 32 (1981), 45-55.

13. M, H $\mathrm{H}_{\mathrm{ALL}}$, JR., The Theory of Groups (Macmillan, 1959).

14. J. Harlander, Minimal Cockcroft subgroups, Glasgow Math. J., to appear.

15. H. Hopf, Fundamentalgruppe und zweite Bettische Gruppe, Comment. Math. Helv. 14 (1941), 257-309.

16. H. Hopf, Beitrage zur Homotopietheorie, Comment. Math. Helv. 17 (1945), 307-326.

17. G. HuCK and S. Rosebrock, A bicombing that implies a sub-exponential isoperimetric inequality, Proc. Edinburgh Math., to appear.

18. J. HuebschmanN, Cohomology theory of aspherical groups and of small cancellation groups. J. Pure Appl. Algebra 14 (1979), 137-143.

19. R. C. LyNDON, Cohomology theory of groups with a single defining relation, Ann. of Math. 52 (1950), 650-665.

20. S. J. PrIDE, Identities among relations of groups presentations, in Proceedings of the Workshop on Group Theory for a Geometrical Viewpoint, Trieste, 1990 (E. Ghys, A. Haefliger and A. Verjovsky, eds., World Scientific Publishing, Singapore 1991), 687-717.

21. S. J. PRIDE, Examples of presentations which are minimally Cockcroft in several different ways, J. Pure Appl. Algebra 88 (1993), 199-204.

Oregon State University

Corvallis

OR 97331, USA 